University for Business and Technology in Kosovo

UBT Knowledge Center

UBT International Conference

2015 UBT International Conference

Nov 7th, 9:00 AM - 5:00 PM

\title{
Hybrid Modelling in System Simulation
}

\author{
Andreas Körner \\ Technische Universität Wien, andreas.koerner@tuwien.ac.at
}

Follow this and additional works at: https://knowledgecenter.ubt-uni.net/conference

Part of the Computer Sciences Commons, and the Digital Communications and Networking Commons

\section{Recommended Citation}

Körner, Andreas, "Hybrid Modelling in System Simulation" (2015). UBT International Conference. 106.

https://knowledgecenter.ubt-uni.net/conference/2015/all-events/106

This Event is brought to you for free and open access by the Publication and Journals at UBT Knowledge Center. It has been accepted for inclusion in UBT International Conference by an authorized administrator of UBT Knowledge Center. For more information, please contact knowledge.center@ubt-uni.net. 


\title{
Hybrid Modelling in System Simulation
}

\author{
Andreas Körner \\ Vienna University of Technology, \\ Institute for Analysis and Scientific Computing, \\ Wiedner Hauptstraße 8-10, 1040 Vienna, Austria \\ andreas.koerner@tuwien.ac.at
}

\begin{abstract}
In times of increasing power and capacity in computer simulation mathematical models are getting more and more important. For different technical applications and in natural science but also in economic systems and management processes appropriate mathematical model descriptions are necessary. Hybrid modelling is a special technique for more complex model descriptions in order to reduce the degree of complexity. In different fields of interest the behavior of a model is dependent on the active state. When the model description is changing from one state to another a so-called state event takes place. State event modelling is the overall term to describe this modelling approach. One state is defined by one dynamic system description and another state is described by the next description. The mathematical environment of the model allows finding the description which is the best matching one in each state. In this sense it is possible to find the most efficient model description for each state and it is not necessary to build up a complicated model structure to cover all cases in one model. Beside the principle effect of the basic structure it is also possible to combine different mathematical modelling techniques for realizing a hybrid model for a certain complex system. In each case a certain mathematical model of the mathematical method can be provided. This is the formal mathematical definition of a multi method approach. In different states different models are simulated and a certain master algorithm is managing the overall administration of the discrimination. The mentioned issues are covered under the overall term hybrid modelling and will be introduced in the corresponding paper.
\end{abstract}

Keywords: Mathematical Modelling, Hybrid Modelling, System Simulation

\section{Introduction}

System simulation is an often used term in the current scientific fields and is as well used for business solutions. Fraunhofer ITWM refers system simulation in the context of simulation models in the automobile sector, see [1]. In this case the term explains the integration of physical attributes in different stages of the development process of a car. In mechatronics the term system simulation covers the field of a 1-D simulation of multidisciplinary systems, as addressed in [2].

In this publication, system simulation is understand as a simulation approach which focuses on the description and simulation of a whole system, e.g. a physical, electrical, mechanical, mechatronical system or process surveyed over time. In this class of simulation approach spatial distributed parameter simulation is not covered, neither diffusion nor relaxation processes nor similar processes simulated by FEM methods or similar.

\section{Modelling Structure and Placement in System Simulation}

\subsection{Hybrid System Modelling}

The term hybrid modelling is within the last 20 years one of the most fashionable terms in the field of modelling and simulation. The meaning of the term is as multifunctional as the fields of science which uses the term in the discipline. According to title of this paper the term hybrid modelling is 
oriented to system simulation and due to this based on dynamic systems. One basic work on dynamic systems and the correlation to hybrid dynamic systems, their modeling and analysis of model is [3]. The basic idea of hybrid modelling is a partition of the whole model in sub-model descriptions which are either partitioned regarding the modelling approach or the modelling technique. With respect to the implantation in a certain simulation environment the model description can be separated for different special cases, where the sum of the special cases are representing the model of the whole system. Benefit of this modeling structure is the cost in the simulation environment.

\subsection{Hybrid Modelling in System Simulation}

System simulation is based on the description of dynamic systems. The solution of this systems are represented by a processing over time. Hybrid dynamic systems are represented by partitioned in different sections where the solution is computed from a sub-model description. This approach is illustrated in Figure 1.

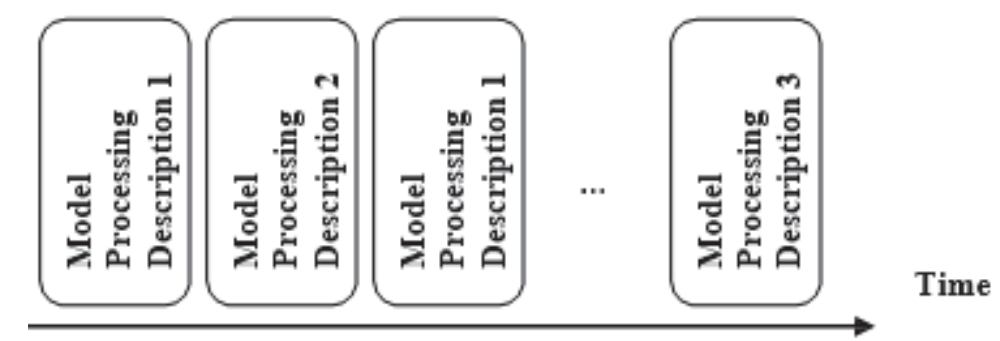

Fig. 1. Illustration of the sequential processing of different sub-model descriptions which performs in total the model processing of a certain system.

One important aspect is the appearance of certain sub-models. At first consideration it can be expected, that a certain number of models are consecutively processed up to the final model of a simulation run, but this is wrong. The model description is partitioned in different sub-models, but the processing over time can allocate one model more often, if the condition for transition is permitting a certain number of requests. Figure 2 is illustrating the relation between the different sub- model descriptions and the transition in between of those.

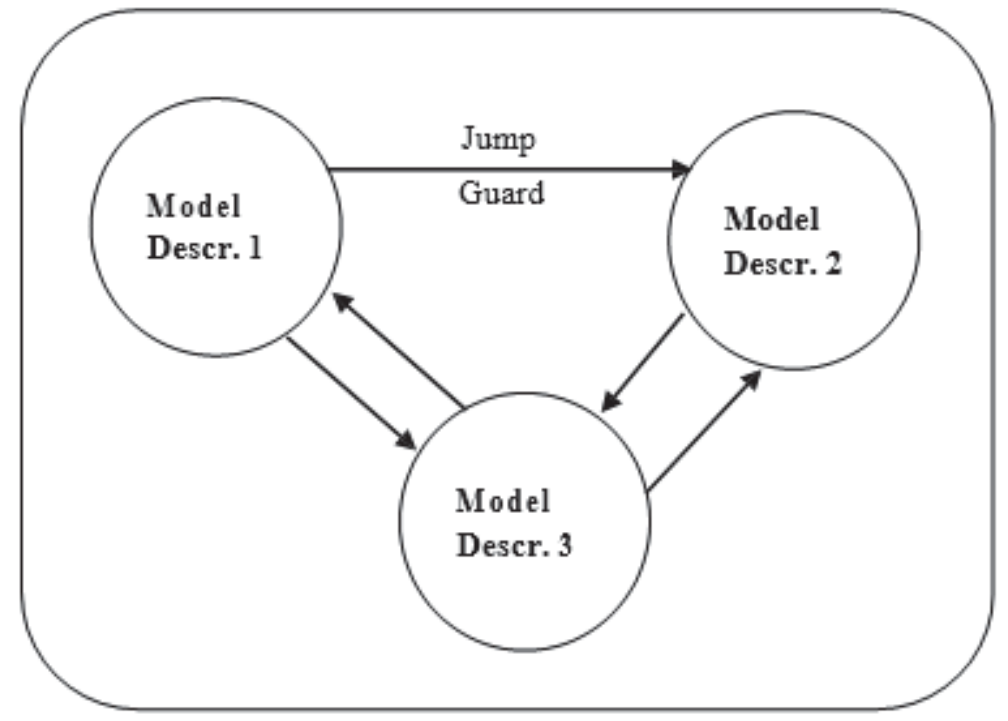

Fig. 2. Illustration of an exemplary Interaction of a Hybrid Model with three different Sub-model Descriptions. 
Figure 1 is representing a time oriented processing domain, whereas Figure 2 focus on the causal relation between the different sub-model descriptions of a hybrid model.

The splitting of the model description in sub-model components is for simulation aspects very important. This offers consideration in distributing different sub-models in different simulation environments which opens the research field of co-simulation and related topics.

\section{Mathematical Aspects and Formalization}

The given structural description in the section before requires a detailed definition of the mathematical model description. Mathematical aspects of the model description are the following:

- Combination of discrete states and continuous dynamic system description

- Discrete Formalization between sub-models: Finite Automaton

- Continuous Formalization of a certain sub-model: Dynamic System - Differential (Algebraic) Equation

- Transition between sub-models: Jumps and Guard region combine the discrete and continuous formalization and link the sub-model to a hybrid model

A mathematical characterization is important to have two interests: To be able to relate the separation of the sub-models w.r.t. the overall model on the one side and the possibility to establish a layer where the comparison of different hybrid model descriptions is possible.

The mathematical formalization of this framework in detail is listed in [4]. This current publication addresses the purpose of hybrid models in system simulation to be able to distinguish between a mathematical model and the simulation model in a specific simulation environment. The model per se covers the abstract description without some considerations of the simulation environment behind. The simulation model consider restriction of the environment and include the first attributes of the numerical simulation.

\section{Summary, Conclusion and Outlook}

The hybrid modelling approach is offering a more efficient way to develop models for complex systems. Of course an overall model description of the whole complex model is in most of the cases available, but for a more efficient model description w.r.t. to simulation aspects the hybrid approach is more powerful.

Simulation environments often restrict the possibilities of modelling and include the considerations regarding numerical mathematics which is interesting for the simulation run of the model but not for the model description. The presented mathematical framework offers a wide range of continuing considerations. On the one side several considerations in the field of the simulation model can be addresses, e.g. co-simulation and multi-method simulation methods, and plural approaches regarding technique in the modelling method allows a multi- method approach in the abstract mathematical environment.

\section{References}

1. Fraunhofer ITWM, http://www.itwm.fraunhofer.de, January 2016

2. Siemens PLM, http://www.plm.automation.siemens.com, January 2016

3. Lygeros J., Tomlin C., Sastry S.: Hybrid Systems: Modeling, Analysis and Control, EECS Instructional Support Group, University of California, Berkeley (2008)

4. Körner A., Breitenecker F.: Approaches for State Event Handling by Simulation Algorithm and via Model Description: 22. Symposium Simulationstechnik, HTW Berlin; 03.-

05.09.2014; in: Proceedings ASIM 201422 Symposium Simulationstechnik, Wittmann J., Deatcu C. (Eds.); ARGESIM/ASIM, 43/2/Vienna, ISBN: 978-3-901608-44-5; p. 219 - 224

(2014). 